\title{
Inteligencia emocional y autocuidado en los \\ estudiantes de Enfermería
}

\author{
Emotional Intelligence and Self-Care in Nursing Students \\ Inteligência emocional e autocuidado em estudantes de \\ Enfermagem
}

Aida Monserrate Macías Alvia aidita.macias@hotmail.com

Tibisay Rincón Rios tibyrin@hotmail.com

Jimmy Geovanny Reyes Pincay jimmy.reyes.p@hotmail.com

Roberth Olmedo Zambrano Santos rzambranosantos@yahoo.es

Recibido enero 2018 / Revisión febrero 2018 / Aceptado 1 de mayo 2018

\section{RESUMEN}

Introducción: La inteligencia emocional incorpora aspectos importantes en las relaciones interpersonales e intrapersonales, la adaptabilidad, los estados de ánimo y las habilidades para el manejo del estrés, que tienen un profundo efecto en el autocuidado de los estudiantes. Objetivo: Determinar el nivel de inteligencia emocional y el autocuidado en los estudiantes de la carrera de enfermería. Materiales y Métodos: Estudio descriptivo, analítico, diseño no experimental, transversal y prospectivo; el método utilizado hipotético-deductivo con un enfoque cuantitativo. Se seleccionaron estudiantes del último bienio de la carrera de enfermería en la Universidad Estatal del Sur de Manabí, con el fin de efectuar un diagnóstico referido al nivel de inteligencia emocional y del nivel de autocuidado. Se recopilaron datos sociodemográficos mediante el uso de encuestas. Los instrumentos aplicados fueron el Test de Inteligencia Emocional y la escala de Agencia de Autocuidado de Esther Gallego aplicado directamente a los estudiantes. Resultados: Se comprobó que los estudiantes presentan un nivel promedio de inteligencia emocional y de autocuidado, con predomino del género femenino, encontrándose además, un bajo rendimiento académico. Conclusión: Se pudo concluir que los estudiantes presentan Nivel Medio de Inteligencia emocional así como también presentan Capacidad de Autocuidado Media

Palabras clave: inteligencia emocional; estudiantes de enfermería; autocuidado

\begin{abstract}
Introduction: Emotional intelligence incorporates important aspects into interpersonal and intrapersonal relationships, adaptability, moods and stress management skills, which have a profound effect on self-care Students. Objective: Determine the level of emotional intelligence and self-care in students of the nursing career. Methods: Descriptive, analytical, non-experimental, cross-cutting and prospective design; the method used hypothetical-deductive with a quantitative approach. Students from the last biennium of nursing degrees were selected at the Southern Manabí State University, during the period October 2018-March 2019, in order to make a diagnosis regarding the level of emotional intelligence and the level of self-care. Sociodemographic data were collected through the use of surveys. The instruments applied were the Emotional Intelligence Test and Esther Gallego's SelfCare Agency scale applied directly to students. Results: Students were found to have an average level of emotional intelligence and self-care, with a predominance of the female gender, and are also found to be poor academic performance. Conclusion: It was concluded that students present Medium Level of Emotional Intelligence as well as present Self-Care Capacity Half
\end{abstract}

Key words: emotional intelligence; nursing students; self-care
AM: Universidad Estatal del Sur de Manabí, Ecuador.

Instituto Tecnológico Superior "Portoviejo", Ecuador.

TR: Universidad del Zulia Facultad de Medicina, Venezuela.

JR: Universidad Estatal del Sur de Manabí, Ecuador.

RZ: Universidad Estatal del Sur de Manabí, Ecuador. 
AM: Universidad Estatal del Sur de Manabí, Ecuador. Instituto Tecnológico Superior "Portoviejo", Ecuador.

TR: Universidad del Zulia Facultad de Medicina, Venezuela.

JR: Universidad Estatal del Sur de Manabí, Ecuador.

RZ: Universidad Estatal del Sur de Manabí, Ecuador.

\section{RESUMO}

Introdução: A inteligência emocional incorpora aspectos importantes em relacionamentos interpessoais e intrapessoais, adaptabilidade, humores e habilidades de gerenciamento de estresse, que têm um efeito profundo no autocuidado Estudantes. Objetivo: Determinar o nível de inteligência emocional e autocuidado nos alunos do carreira de enfermagem. Métodos: Design descritivo, analítico, não experimental, cross-cutting e prospectivo; o método usou hipotéticodedutivo com uma abordagem quantitativa. Estudantes do último biênio de enfermagem foram selecionados na Universidade Estadual de Manabí do Sul, durante o período outubro de 2018 a março de 2019, a fim de fazer um diagnóstico sobre o nível de inteligência emocional e do nível de autocuidado. Os dados sociodemográficos foram coletados através do uso de inquéritos. Os instrumentos aplicados foram o Teste de Inteligência Emocional e a escala da Agência de Autocuidado de Esther Gallego aplicada diretamente aos alunos. Resultados: Verificou-se que os alunos têm um nível médio de inteligência emocional e autocuidado, com predomínio do sexo feminino, e também são considerados baixo desempenho acadêmico. Conclusão: Concluiu-se que os alunos apresentam Nível Médio de Inteligência Emocional, bem como apresentam Meia-Chave de Capacidade de Autocuidado: Inteligência Emocional, Estudantes de Enfermagem, Autocuidado

Palavras-chave: inteligência emocional; estudantes de enfermagem; autocuidado

\section{INTRODUCCIÓN}

$\mathrm{E}$ $\mathrm{n}$ el mundo moderno, los estudiantes sienten dificultad para controlar sus emociones y con ello para enfrentar diversas situaciones en su vida académica y personal. El éxito en el mundo académico se puede predecir más con medidas emocionales. Un estudiante debe ser emocionalmente inteligente $\mathrm{y}$ mentalmente sano para que pueda lograr buenos resultados académicos. Se espera que los estudiantes de enfermería muestren emociones que transmitan cariño, comprensión y compasión hacia los pacientes mientras regulan sus propios sentimientos. Solo estudiantes emocionalmente competentes pueden resistir el problema aplicando los componentes de la inteligencia emocional como son autoconciencia, autorregulación, auto motivación y habilidades interpersonales (1)

Son más de 30 años de investigación y estudio del concepto, y hoy día no existe un consenso para una definición única de Inteligencia Emocional (IE). Existen múltiples instrumentos de pruebas $\mathrm{y}$ diferentes opiniones acerca de su medición. También continúan existiendo relativamente pocos estudios de IE específicos en los estudiantes de enfermería. Para los fines de esta investigación, se definió a la IE como la capacidad de controlar las propias emociones e influir en las emociones de los demás. Los estudios se centran en la medición de uno o más de los siguientes ocho rasgos o habilidades específicos relacionados con la IE: liderazgo, salud, reflexión, desempeño de los estudiantes de enfermería, comportamiento ético, cuidado, pensamiento crítico y retención / satisfacción laboral (2).

La definición de IE como habilidades que permiten la capacitación en competencias específicas que pueden aplicarse directamente a un campo especializado, se conceptualiza como una habilidad que se puede enseñar, aprender y cambiar, para abordar los aspectos disfuncionales de la relación médicopaciente. Por este motivo, la enseñanza de la IE debe ser una prioridad en el campo de la educación médica para facilitar esta relación en el futuro. Del mismo modo, los estudiantes de enfermería deben tomar 
conciencia de sus propios sentimientos y pensamientos frente a los pacientes y sus familiares. Esto requiere que adquieran IE y habilidades de pensamiento crítico, en base a su preparación para brindar una práctica de enfermería competente y ayudarlos a adaptarse al entorno clínico (3).

Para tener la IE, los estudiantes de enfermería necesitan una fuerza interior para canalizar y dirigir su pensamiento inconsciente para el mayor bien de los pacientes bajo su cuidado y sus componentes estudiantiles (4).

Los estudiantes de enfermería, hoy en día, tienen la responsabilidad de mantener su propia salud, así como la de sus semejantes. No obstante, frecuentemente colocan el cuidado personal en el último lugar de sus responsabilidades. Las prácticas de autocuidado se definen como las actividades que las personas maduras inician y realizan, dentro de los plazos, en su propio beneficio con la finalidad de mantener la vida, el funcionamiento saludable, el desarrollo personal continuo y el bienestar, a través del cumplimiento de los requisitos conocidos para el desarrollo funcional. En otras palabras, las prácticas de autocuidado se pueden utilizar para mantener y promover la propia salud. Los estudiantes tradicionalmente tienen niveles bajos de autocuidado, lo cual es evidente en muchas dimensiones específicas (5).

De acuerdo a la Organización Mundial de la Salud (OMS), el autocuidado es la capacidad de las personas, las familias y las comunidades para promover, mantener la salud, prevenir enfermedades y hacer frente a las enfermedades con o sin el apoyo de un proveedor de atención médica. Además abarca varios problemas, tales como higiene, nutrición, estilo de vida, factores ambientales $\mathrm{y}$ socioeconómicos. Sin embargo, su promoción está dirigida a la capacitación de individuos, familias y comunidades para la toma de decisiones y tener el potencial de mejorar la eficiencia y la equidad de los sistemas de salud (6).

El Ministerio de Salud Pública del Ecuador tiene como finalidad optimizar recursos para realizar proyectos investigativos, lograr un impacto positivo en la calidad de la misma y mejorar la eficiencia para enfatizar en el autocuidado de sus enfermedades y su apariencia personal, coordinar los esfuerzos de todos los actores del sistema de salud para lograr los objetivos del Buen Vivir (7).

El autocuidado puede evaluarse de forma efectiva utilizando los requisitos universales de Orem. Las áreas incluidas en este marco incluyen: aire, alimentos, líquidos, eliminación, actividad y descanso, interacción social, normalidad y prevención de riesgos. Todas estas áreas contribuyen a un retrato de salud integral del individuo. Mientras aprenden a cuidar a otros, los estudiantes de enfermería a menudo no se cuidan a sí mismos, debido a la mala gestión de las conductas de cuidado personal que contribuyen a la insatisfacción laboral, el agotamiento académico y el desgaste. Es por eso que existen tendencias descendentes de comportamientos de cuidado de sí mismos, a medida que los estudiantes asumen una mayor responsabilidad por el cuidado de los demás $(5,8)$.

La IE se ha asociado con resultados positivos para los estudiantes de enfermería en relación a su autocuidado en forma general. Una mayor IE se asocia con el bienestar personal y la gestión adecuada del estrés. El manejo de las emociones de los demás se asoció positivamente con un mayor rendimiento académico entre los estudiantes, un liderazgo de enfermería en su contexto estudiantil y un rendimiento de la práctica más sólidos y una mayor seguridad del paciente. Si bien existe un creciente cuerpo de evidencia sobre la IE 
de los estudiantes de enfermería, consta una evidencia mínima sobre ella a lo largo del tiempo durante los programas de preinscripción. Sin embargo, la educación de enfermería previa al registro contribuye al fortalecimiento de la IE de los estudiantes con el tiempo (9).

Los estudiantes se forman en la universidad para cuidar a otros, pero no para cuidarse a sí mismos. Las universidades desempeñan un papel destacado en la sociedad en la que están integradas. Su objetivo es desarrollar el conocimiento, son el escenario para la formación profesional de ciudadanos y personas responsables $\mathrm{y}$, a través de la educación, la investigación y la responsabilidad social, promueven el desarrollo y los cambios en varios dominios nacionales e internacionales (10).

La IE y el autocuidado son vitales para los estudiantes de enfermería para aliviar los efectos perjudiciales que el estrés ocasiona debido al entorno asistencial que cambia constantemente y puede tener consecuencias devastadoras para las enfermeras y enfermeros así como también para las personas bajo su cuidado. Es por este motivo que ha surgido un creciente interés en la atención en todas las personas, los líderes de los procesos de enfermería en las organizaciones o establecimientos de atención médica, están prestando más atención a la cultura de entornos de práctica que apoyan principios centrados similares para el personal de enfermería, interprofesionales y otros empleados (11).

Es importante determinar el nivel de IE entre los estudiantes de enfermería y su relación con el autocuidado como primer paso antes de desarrollar y evaluar las intervenciones para mejorar estas habilidades. Esto es especialmente significativo, considerando la importancia de la educación para promover el desarrollo emocional y social de los estudiantes en todos los niveles educativos.

Desde un punto de vista científico, la IE es la capacidad de percibir con precisión sus propias emociones y las de los demás; comprender las señales que envían las emociones sobre las relaciones y manejar tus propias emociones y las de los otros. No necesariamente incluye las cualidades (como el optimismo, la iniciativa y la confianza en sí mismo) que algunas definiciones populares le atribuyen (12).

La falta de preparación emocional puede conllevar al estrés $\mathrm{y}$, en última instancia, al agotamiento, disminución del afrontamiento y la resiliencia por lo que los estudiantes de enfermería, abandonan el entorno de los estudios universitarios o incluso la profesión. Kaur, Sambasivan y Kumar y sus colaboradores encontraron que la IE puede reducir los efectos del agotamiento en los profesionales en formación y mejorar sus sentimientos de pertenencia hacia sus estudios universitarios.

Se refiere la IE a la capacidad de identificar, expresar, comprender y regular las emociones, ya sea de manera negativa o positiva, en uno mismo y en los demás (13). Implica poseer creatividad, la capacidad de desempeñarse a un nivel óptimo al completar tareas, resistencia y resiliencia, con capacidad de persistir a pesar de los contratiempos y las fallas. Las habilidades emocionales se aprenden durante la niñez y la infancia, pero además se pueden desarrollar y lograr cambios más adelante con la edad, la experiencia y la influencia de buenos modelos y mentores. Un individuo con una IE más alta que el promedio, es generalmente más exitoso para satisfacer las demandas y presiones ambientales (14).

Un grupo de docentes y estudiantes investigadores de la Universidad Estatal del Sur de Manabí, investigaron a 
profundidad lo que sucede con el autocuidado de los estudiantes de enfermería. De acuerdo a los precedentes anteriormente mencionados, se están realizando estudios respecto al tema. La atención plena implica desarrollar una intencionalidad, conciencia abierta y aceptadora, permitiéndose responder en lugar de reaccionar ante situaciones.

La Universidad Estatal del Sur de Manabí, es una institución en la que se encuentran estudiantes de diversos cantones y provincias, por lo tanto, la presente investigación se justifica porque uno de los problemas esenciales a nivel de los estudiantes de enfermería es la tendencia al estrés académico y la afectación de su cuidado personal que podría contribuir a muchos problemas físicos y mentales. Sin embargo, hay poca evidencia empírica sobre la asociación entre la IE y el autocuidado en los estudiantes de enfermería. El objetivo general de la presente investigación será determinar la IE y el autocuidado en los estudiantes de la carrera de enfermería de la Universidad Estatal del Sur de Manabí.

\section{MATERIALES Y MÉTODOS}

$\mathrm{E}$ n esta investigación se realizó un estudio descriptivo, analítico, de corte transversal, prospectivo. El método utilizado fue hipotético-deductivo, con un enfoque cuantitativo y un universo constituido por 313 estudiantes. Se aplicó la técnica de cálculo de la muestra aleatorio estratificado, con el uso del estadístico SPSS, estipulando un intervalo de confianza del $95 \%$ y un margen de error de $5 \%$. El total de la muestra para el estudio fue de 187 estudiantes de quinto al octavo semestre de la carrera de enfermería de la Universidad Estatal del Sur de Manabí, durante el periodo comprendido Octubre 2018-Marzo 2019.

El instrumento aplicado fue la escala de Agencia de Auto Cuidado de Esther Gallego, la cual consta de 24 ítems, y el Test de Inteligencia Emocional (Trait Meta-Mood Scale-24) que consta de 24 ítems y 5 alternativas de respuestas. Los datos obtenidos fueron tabulados con el software SPSS.

Criterios de inclusión. Se seleccionaron estudiantes mayores de 18 años de edad, sin distingo de etnia, género o procedencia, aceptación y firma del consentimiento informado. Criterios de exclusión. Estudiantes que manifestaron su deseo de no participar en la investigación, los inasistentes o inactivos en el período en que se aplicó el instrumento.

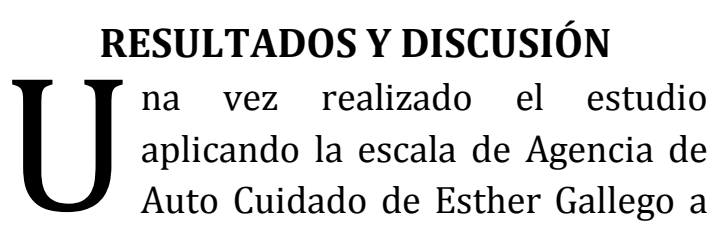
la muestra de 187 estudiantes del quinto al octavo semestres de la carrera de enfermería de la Universidad Estatal del Sur de Manabí, se puede detallar lo siguiente: 
Tabla 1. Distribución de elección expresada en porcentaje del perfil factor sociodemográfico del total de la población

\begin{tabular}{|c|c|c|c|}
\hline & Parámetros & Frecuencia & Porcentaje \\
\hline & 18 & 18 & 9,6 \\
\hline & 19 & 56 & 29,9 \\
\hline \multirow[t]{3}{*}{ Edad } & 20 & 79 & 42,2 \\
\hline & 21 & 23 & 12,3 \\
\hline & 22 & 11 & 5,9 \\
\hline \multirow{4}{*}{ Sexo } & Femenino & 99 & 52,9 \\
\hline & Masculino & 88 & 47,1 \\
\hline & Soltero & 129 & 69 \\
\hline & Comprometido & 7 & 3,7 \\
\hline \multirow{4}{*}{ Estado civil } & Casado & 17 & 9,1 \\
\hline & Unión libre & 32 & 17,1 \\
\hline & Separado & 2 & 1,1 \\
\hline & Viudo & 0 & 0 \\
\hline \multirow{3}{*}{ Procedencia } & Urbana & 123 & 65,8 \\
\hline & Rural & 64 & 34,2 \\
\hline & Casa de los padres & 108 & 57,8 \\
\hline \multirow{4}{*}{ Conviven } & Algún familiar & 26 & 13,9 \\
\hline & Arrienda & 41 & 21,9 \\
\hline & Otros & 12 & 6,4 \\
\hline & No & 129 & 69 \\
\hline \multirow[t]{3}{*}{ Número de hijos } & 1 & 53 & 28,3 \\
\hline & Más de 2 & 5 & 2,7 \\
\hline & Católico & 133 & 71,1 \\
\hline \multirow{5}{*}{ Religión } & Evangélico & 12 & 6,4 \\
\hline & Ateo & 2 & 1,1 \\
\hline & Otros & 40 & 21,4 \\
\hline & Mestiza & 98 & 52,4 \\
\hline & Montubio & 78 & 41,7 \\
\hline \multirow[t]{3}{*}{ Etnia } & Blanca & 2 & 1,1 \\
\hline & Negro & 1 & 0,5 \\
\hline & Otros & 8 & 4,3 \\
\hline
\end{tabular}

Fuente: Instrumento aplicado

Se observó que la edad que predominó en el estudio fue de 20 años con el 42,2\%. Una investigación realizada por Deeksha Sharma, demostró que la Inteligencia emocional total aumentó con la edad. La competencia emocional disminuyó desde la edad adulta temprana (17-23 años), hasta la mediana edad (24-34 años) y luego aumentó a la edad madura (35-60). La madurez era máxima para la edad 
madura, mientras que la competencia y la sensibilidad eran máximas para la mediana edad. El sexo femenino predominó con el 52,9\%, resultado comparable con lo expuesto por los investigadores Gartzia, Aritzeta, Balluerka y Heredia, donde demostraron que las mujeres con rasgos expresivos presentaron puntuaciones más altas en el desarrollo de la IE.

Los estudiantes que no tenían pareja representan el 70,1\%, en relación a las otras dimensiones del estado civil.

La religión católica predominó con el $71,1 \%$ en relación a las otras religiones. Los resultados presentados por Masoumeh determinaron la relación entre la IE, con el afrontamiento religioso y la salud mental de los estudiantes de la
Universidad de Ciencias Médicas de Mazandaran. Según los resultados de este estudio, existe una correlación directa entre el afrontamiento religioso positivo y la IE. Por lo tanto, el fortalecimiento del manejo religioso puede promover la IE que es un componente de la salud mental.

Se evidencia que dos etnias predominan en el estudio, pero con un rango mayor ser mestizo en relación a montubio con el 52,4\%. Según la investigación realizada por Zahrasari L. Dewi que tuvo como propósito verificar las competencias de la IE de tres grupos étnicos diferentes en Indonesia, los resultados demostraron que existen efectos principales significativos de la etnicidad y el género, así como el efecto de interacción en las competencias de la IE.

Tabla 2. Distribución de elección expresada en porcentaje del nivel de inteligencia emocional del total de la población

\begin{tabular}{cccc}
\hline Orden & Alternativa & F & $\mathbf{\%}$ \\
\hline A & Necesita ayuda & 49 & $26,2 \%$ \\
B & Nivel promedio de inteligencia & 82 & $43,8 \%$ \\
C & Inteligencia emocional superior & 56 & $29,9 \%$ \\
\hline Total & & 187 & 100 \\
\hline
\end{tabular}

Fuente: Instrumento aplicado

Tabla 3. Distribución de elección expresada en porcentaje del nivel de agencia de autocuidado del total de la población

\begin{tabular}{cccc}
\hline Orden & Alternativa & F & \% \\
\hline A & Baja & 53 & $28,3 \%$ \\
B & Media & 77 & $41,2 \%$ \\
C & Alta & 57 & $30,5 \%$ \\
\hline Total & & 187 & 100 \\
\hline
\end{tabular}

Fuente: Instrumento aplicado 
En relación al autocuidado, se encontró que los estudiantes presentan un Nivel Medio de Autocuidado con el 41,2\%, sin embargo, llama la atención que existe un porcentaje representativo de bajo nivel de autocuidado $(28,3 \%)$ siendo carrera de salud no debería evidenciarse esta problemática en los estudiantes de la carrera de enfermería porque se están formando como modelos, promotores y protectores de la salud.

Tabla 4. Distribución de elección expresada en porcentaje para los factores de la inteligencia emocional sobre la afectación del rendimiento académico

\begin{tabular}{llrc}
\hline \multicolumn{1}{c}{ VARIABLES } & & \% \\
\hline Factores psicológicos & Confianza en sí mismo. & 59 & 31,6 \\
& Hábitos de estudio. & 48 & 25,7 \\
& Control y manejo de la frustración. & 39 & 20,9 \\
& Capacidad de aprovechamiento. & 41 & 21,8 \\
Factores fisiológicos & Visión. & 62 & 38,1 \\
& Audición. & 54 & 28,9 \\
& Estado general de salud. & 71 & 31,0 \\
Factores sociológicos & Importancia que se le da a la educación en el hogar. & 38 & 20,3 \\
& Grado de escolaridad de los padres. & 50 & 26,7 \\
& $\begin{array}{l}\text { Nivel socioeconómico. } \\
\text { Factores pedagógicos }\end{array}$ & $\mathbf{9}$ & $\mathbf{5 3 , 0}$ \\
& $\begin{array}{l}\text { La pedagogía basada fundamentalmente en } \\
\text { memoria. }\end{array}$ & 60 & 32,1 \\
& $\begin{array}{l}\text { Recurrir demasiado a las calificaciones bajas, como } \\
\text { arma para que los alumnos trabajen. }\end{array}$ & 63 & 33,7 \\
& $\begin{array}{l}\text { Malas metodologías en el aprendizaje de la lecto- } \\
\text { escritura. }\end{array}$ & 64 & 34,2 \\
\hline
\end{tabular}

Fuente: Instrumento aplicado

De acuerdo a estos resultados, se observa que existe una gran afectación en el rendimiento académico de los estudiantes de enfermería posiblemente debido a que se identificó un porcentaje representativo de estudiantes con nivel bajo de autocuidado y de IE. La variable que se observa de mayor impacto es el nivel socioeconómico de los estudiantes de enfermería. Algunos investigadores encontraron de acuerdo a estudios realizados, que la disminución de la inteligencia emocional interfiere en tener un adecuado autocuidado y de afrontamiento y eleva el estrés lo cual conlleva y está altamente correlacionada, con el rendimiento académico deteriorado.

\section{CONCLUSIONES}

A tendiendo al objetivo general de la presente investigación que fue determinar el nivel de inteligencia emocional $y$ el autocuidado en los estudiantes de la carrera de enfermería se concluye que: Los participantes presentan un promedio de Inteligencia Emocional (Media), las percepciones de la inteligencia emocional por parte de estudiantes de 
enfermería pueden representar una estrategia de afrontamiento eficaz en una situación en la que están implicadas las emociones. En la profesión de la salud, una ocupación con un alto nivel de trabajo emocional, es importante identificar los tipos de inteligencia emocional para establecer una estrategia de afrontamiento eficaz, que puede tener un efecto positivo en el desempeño individual y colectivo de los miembros del equipo sanitario y en consecuencia de la organización. Estos resultados podrían indicar un riesgo ya que el promedio detectado podría decaer de moderado a baja. Por lo tanto, es necesario desde el currículo de enfermería promover y fortalecer la IE en los futuros profesionales.

\section{REFERENCIAS}

1. Fallahzadeh. The relationship between emotional intelligence and academic achievement in medical science students in Iran. Procedia - Soc Behav Sci. 2011; 30: 1461-1466

2. Michelangelo $L$. The overall impact of emotional intelligence on nursing students and nursing. Asia-Pac J Oncol Nurs. 2015; 2(2): 118-124

3. Raghubir $A E$. Emotional intelligence in professional nursing practice: A concept review using Rodgers's evolutionary analysis approach. Int J Nurs Sci. 2017; 5(2):126-130

4. Goudarzian $A H$, Nesami MB, Sedghi $P$, Gholami M, Faraji M, Hatkehlouei MB. The effect of self-care education on emotional intelligence of iranian nursing students: A quasi-experimental study. J Relig Health. 2018

5. Ashcraft PF, Gatto SL. Care of self in undergraduate nursing students: A pilot study. Nurs Educ Perspect. 2017; 36(4): 255-256
6. Organization WH, Asia RO for S-E. Selfcare for health [en línea]. WHO Regional Office for South-East Asia; 2014 [citado 201723 noviembre]. Disponible en: https://apps.who.int/iris/handle/10665/205887

7. Ministerio de la Salud Pública de Ecuador. Modelo de Atención Integral del Sistema Nacional de Salud 2010. Disponible en http://instituciones.msp.gob.ec/somossalud/im ages/documentos/guia/Manual

8. Rocha M, González C, Juárez E, Olvera A, Márquez $A$, Rosales $S$ et al Agencia de autocuidado en alumnos de nuevo ingreso en la unidad académica Multidisciplinaria Zona Media. Cienc. enferm. [Internet]. 2015 [citado 2017 octubre 23] ; 21( 1 ): 103-113. doi.org/10.4067/S0717-95532015000100010.

9. Foster K, Fethney J, McKenzie H, Fisher M, Harkness E, Kozlowski D. Emotional intelligence increases over time: A longitudinal study of Australian pre-registration nursing students. Nurse Educ Today. 2017; 55: 65-70

10. Siappo CLG, Núñez YR, Cabral IE, Siappo CLG, Núñez YR, Cabral IE. Nursing students' experiences in selfcare during training process in a private university in Chimbote, Peru. Esc Anna Nery. 2016; 20(1):17-24

11. Ranjbar M, Bahrami MA, Hadizadeh F, Arab M, Nasiri S, Amiresmaili M, et al. Nurses' emotional intelligence impact on the quality of hospital services. Iran Red Crescent Med J. 2015; 14 (12):758-763.

12. Allen DE, Ploeg J, Kaasalainen S. The relationship between emotional intelligence and clinical teaching effectiveness in nursing faculty. J Prof Nurs Off $\mathrm{J}$ Am Assoc Coll Nurs. 2012; 28(4):231-240

13. Birks YF, Watt IS. Emotional intelligence and patient-centred care. J R Soc Med. 2015; 100(8):368-374

14. Cejudo M. La Educación Emocional en la familia. En: Orejudo S, Royo F, Soler J, Editores. Inteligencia Emocional y Bienestar Reflexiones, experiencias profesionales e investigaciones. España: Universidad de Zaragoza; 2014.p 672-686

- Los autores expresan no poseer conflictos de interés.

- Este estudio fue realizado a través de financiamiento propio.

- Apegado a la norma bioética según el tratado de Helsinki 